phys. stat. sol. (b) 44, 759 (1971)

Subject classification : 6 and $20.1 ; 10 ; 22.5 .2$

Max-Planck-Institut |ür Festkörperforschung, Stuttgart (a), and

Physikalisches Institut der Universität Freiburg (b)

\title{
Low Lying Resonant Band Modes in CsBr and CsI Crystals Doped with $\mathrm{In}^{+}$and $\mathrm{Tl}^{+}$
}

\author{
By \\ W. Pretrt (a) and E. Siep (b)
}

\begin{abstract}
Measurements of the far infrared absorption due to $\mathrm{In}^{+}$and $\mathrm{Tl}^{+}$impurities in $\mathrm{CsBr}$ and CsI single crystals yielded low frequency resonances with narrow linewidths at $11.0 \mathrm{~cm}^{-1}$ in CsBr: $\mathrm{In}^{+}, 12.0 \mathrm{~cm}^{-1}$ in $\mathrm{CsI}: \mathrm{In}^{+}, 14.1 \mathrm{~cm}^{-1}$ in $\mathrm{CsI}: \mathrm{Tl}^{+}$, and $16.95 \mathrm{~cm}^{-1}$ in $\mathrm{CsBr}: \mathrm{Tl}^{+}$. The frequencies of the resonant band modes in the $\mathrm{Tl}^{+}$doped crystals shift to higher values with increasing temperature. Using a model of the perturbed crystal which involves the mass defect and the change of the central force constants between the defect and its eight nearest neighbours, the force constant change is fitted to the various resonance frequencies. The resulting linewidths are compared with the experimentally determined values.
\end{abstract}

Messungen der Fern-Infrarot-Absorption von $\mathrm{In}^{+}$und $\mathrm{Tl}^{+}$Defekten in CsBr- and CsJEinkristallen ergaben niederfrequente und schmalbandige Resonanzen bei $11,0 \mathrm{~cm}^{-1}$ in $\mathrm{CsBr}: \mathrm{In}^{+}, 12,0 \mathrm{~cm}^{-1}$ in $\mathrm{CsJ}: \mathrm{In}^{+}, 14,1 \mathrm{~cm}^{-1}$ in $\mathrm{CsJ}: \mathrm{Tl}^{+}$und $16,95 \mathrm{~cm}^{-1}$ in $\mathrm{CsBr}: \mathrm{Tl}^{+}$. Die Frequenzen der resonanten Bandschwingungen in den $\mathrm{Tl}^{+}$-dotierten Kristallen verschieben sich mit zunehmender Temperatur zu höheren Werten. Hit Hilfe eines Modells des gestörten Kristalls, welches den Massendefekt und die Änderung der zentralen Kraftkonstanten zwischen dem Defekt und seinen acht nächsten Nachbarn berücksichtigt, wurde die Kraftkonstantenänderung an die verschiedenen Resonanzfrequenzen angepaBt. Die hieraus resultierenden Linienbreiten wurden mit den experimentell ermittelten Werten verglichen.

\section{Introduction}

Various investigations concerning the lattice dynamics of defect crystals have shown that particular atomic impurities in alkali halide crystals exhibit resonances whose frequencies lie in the continuum of the long wavelength acoustic phonons. Resonant lattice modes in which an impurity vibrates with a different amplitude than does the replaced ion are associated with an electric dipole moment and can be directly observed as narrow absorption lines in the far infrared. Such defect modes result from either a heavy impurity or, which is more reasonable in most cases, from a weak coupling between the defect ion and the lattice. The linewidth of a resonant mode is finite even at zero temperature due to scattering of phonons of the unperturbed lattice by the impurity. Therefore the relative linewidth of a resonant mode is proportional to the phonon density of states. In [1] to [6] full details of the properties of low frequency resonant modes are given.

So far much work has been done on the far infrared absorption of doped alkali halides with sodium chloride structure. In this paper we present results concerning $\mathrm{In}^{+}$and $\mathrm{Tl}^{+}$impurities in cesium bromide and cesium iodide host lattices both of which crystallize with the cesium chloride structure. Preliminary measurements of the impurity induced absorption of CsI:Tl+ have already been published elsewhere [7]. 


\section{Experimental}

\subsection{Technique}

The absorption measurements were made using a commercial RIIC Michelson interferometer, FS 720, and a Fourier transform computer, FTC 100. The $\mathrm{Tl}^{+}$doped single erystals were grown from u.p. material by the Czochralski technique in air. The $\mathrm{Tl}^{+}$concentrations of these samples were determined by colorimetric chemical analysis [8]. The $\mathrm{In}^{+}$doped samples were grown with the vacuum Bridgman method. ${ }^{1}$ ) Their impurity concentrations are only approximately known. The samples were mounted in a cryostat and were cooled by helium exchange gas in thermal contact with liquid helium or nitrogen. The temperature was adjusted by electrical heating of the exchange gas chamber and was measured using a calibrated Allen Bradley carbon resistor of $100 \Omega$ nominal resistance. The coefficient of additional absorption was determined by the usual manner of comparing the transmission spectra of a doped and an undoped sample of equal thicknesses.

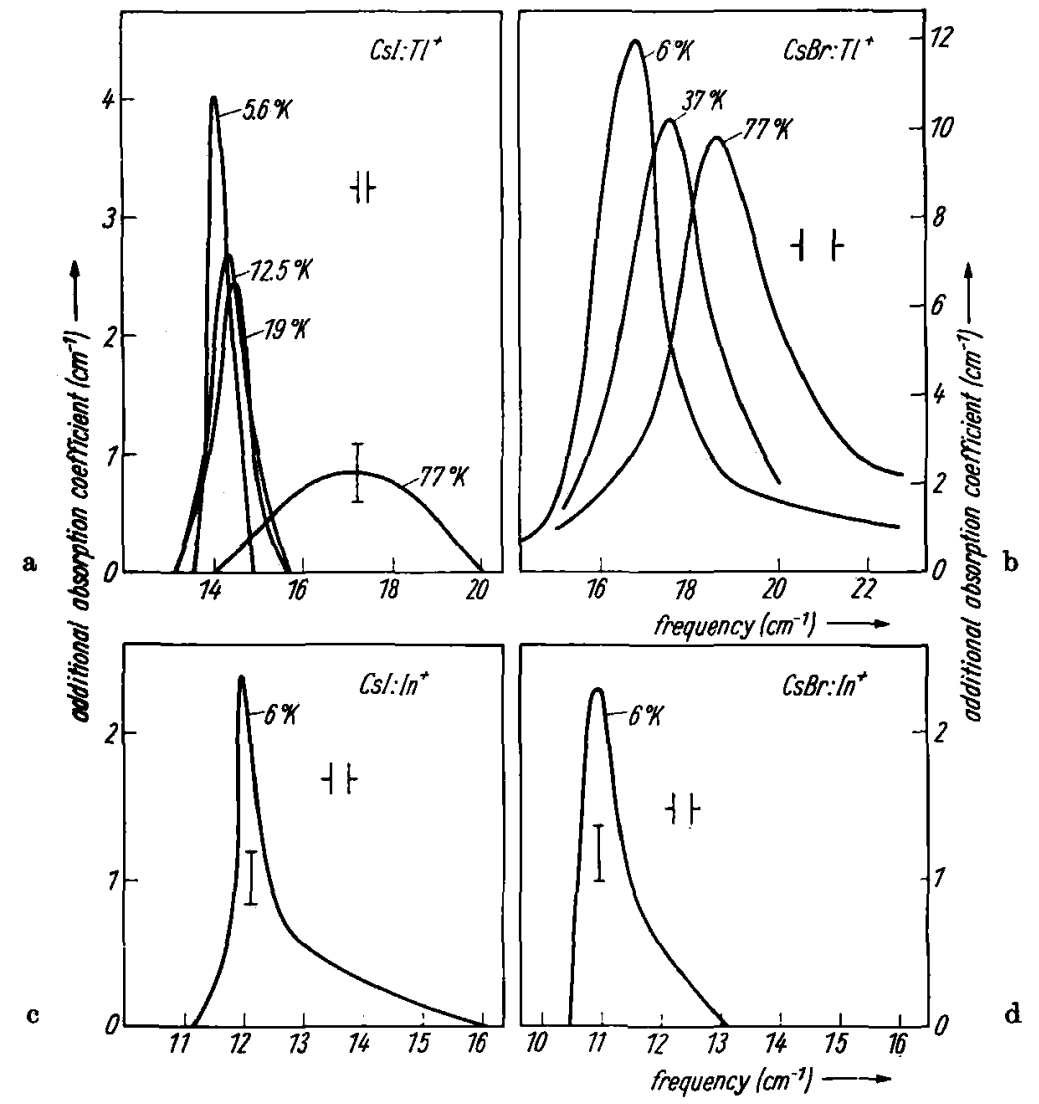

Fig. 1. Resonant band mode alsorption. Impurity concentrations:

CsI:T1 $1.5 \times 10^{-3} \mathrm{~mol}^{+}, \mathrm{CsBr}_{\mathrm{T}} \mathrm{T}^{+} 6.4 \times 10^{-2} \mathrm{~mol} \%, \mathrm{CsI}: \mathrm{In}^{+}$and $\mathrm{CsBr}: \mathrm{In}^{+} \approx 10^{-3} \mathrm{~mol}^{\circ}[16]$

1) These crystals were grown by Dr. K. MAIER at the University of Frankfurt and placed at our disposal. 


\subsection{Results and discussion}

The experimental results are shown in Fig. 1 where the curves of the additional absorption due to the impurities are given. In all four systems resonant band modes were found whose frequencies lie close together between 11.0 and $16.95 \mathrm{~cm}^{-1}$. The resonance frequencies and the linewidths (full width at half maximum absorption) at about $6^{\circ} \mathrm{K}$ are listed in Table 1 column (a) and (b), respectively. The given values of the linewidths are corrected to eliminate the instrumental resolution according to Kirby et al. [6]. All absorption curves are more or less asymmetric. The asymmetry of the $\mathrm{Tl}^{+}$modes is largely due to the increasing phonon density of states. It becomes nearly symmetrical upon dividing by the density of states calculated from the breathing shell model $[9,10]$. The origin of the asymmetry of the $\mathrm{In}^{+}$resonances is not quite clear. Though natural mixtures of isotopes have been used for doping, a hidden isotope splitting is not to be expected, because the abundance of the lighter In isotope amounts to only $4.23 \%$. Also a ground state splitting caused by off-centre equilibrium positions of the $\mathrm{In}^{+}$ion cannot be concluded from the absorption curves because the temperature during the measurement was too high. Tentatively the asymmetry might be assigned to dislocations interacting with the volume defect $\mathrm{In}^{+}$. The small sized impurity has its stable equilibrium position in the compressional part of a dislocation, hence the frequency of impurities on such sites would be increased thus causing the high frequency shoulder of the line. A side band of the resonant mode in $\mathrm{NaCl}: \mathrm{Cu}^{+}$could be explained with similar arguments [11].

The weak coupling of the defect ions might be qualitatively explained by the following arguments:

(i) The ionic radii of $\mathrm{In}^{+}$and $\mathrm{Tl}^{+}$are, respectively, about 22 and $17 \%$ smaller than that of the substituted $\mathrm{Cs}^{+}$ion [12]. Moreover the polarizability of $\mathrm{Tl}^{+}$ is larger than that of $\mathrm{Cs}^{+}[13]$.

(ii) The $\mathrm{Br}^{-}$and $\mathrm{I}^{-}$have ionic radii approximately 6 and $8 \%$, respectively, less than one half of the lattice constant of the corresponding cesium halide. Thus, no substantial inward relaxation of the neighbouring ions of the impurities is to be expected. The eight nearest neighbours located on the corners of a cube form an ample cage around one impurity.

\section{Table 1}

(a) Resonance frequencies, (b) experimental determined linewidths, (c) theoretical linewidths, (d) fractional change of the nearest neighbour force constants. All frequencies given in wavenumbers $\left(\mathrm{cm}^{-1}\right)$

\begin{tabular}{c|c|c|c|c|c}
\hline $\begin{array}{c}\text { host } \\
\text { lattice }\end{array}$ & defect & $\begin{array}{c}(\mathrm{a}) \\
\omega_{\mathrm{r}}\end{array}$ & $\begin{array}{c}(\mathrm{b}) \\
\Delta \omega_{\text {exp. }}\end{array}$ & $\begin{array}{c}(\mathrm{c}) \\
\Delta \omega_{\text {th. }}\end{array}$ & $\begin{array}{c}(\mathrm{d}) \\
\Delta f / f\end{array}$ \\
\hline \multirow{3}{*}{$\mathrm{CsBr}$} & $\mathrm{In}^{+}$ & 11.0 & 0.64 & 0.15 & -0.976 \\
\hline \multirow{2}{*}{$\mathrm{CsI}$} & $\mathrm{Tl}^{+}$ & 16.95 & 1.5 & 0.76 & -0.921 \\
\cline { 2 - 6 } & $\mathrm{In}^{+}$ & 12.0 & 0.26 & 0.3 & -0.956 \\
\cline { 2 - 6 } & $\mathrm{TI}^{+}$ & 14.1 & 0.5 & 0.6 & -0.925
\end{tabular}


(iii) The uv absorption spectrum of the $\mathrm{Tl}^{+}$centre in CsI caused by electronic transitions is known to display some anomalies compared with the spectrum of $\mathrm{Tl}^{+}$in other alkali halides [14]. There are more absorption bands partly with different temperature dependence than expected for an (ns) ${ }^{2}$-ion in a crystal field of $\mathrm{O}_{\mathrm{h}}$ symmetry. Recently Maier observed that the uv absorption of $\mathrm{CsBr}: \mathrm{Tl}^{+}$ and also that of the isoelectronic ion $\mathrm{In}^{+}$in $\mathrm{CsI}$ and $\mathrm{CsBr}$ reveals a similar behaviour as $\mathrm{CsI}: \mathrm{Tl}^{+}[15,16]$. Moreover he clarified these anomalies assuming LCAO-molecular orbitals constructed from the electronic wave functions of the impurity and its neighbouring lattice ions instead of treating the problem with simple crystal field theory. This result indicates that the chemical bonding is rather similar for both impurities in both host lattices. This covalent bond might increase the negative charge density between the impurity and its neighbours and hence shield the Coulomb interaction.

The absorption curves of the $\mathrm{Tl}^{+}$resonances have been measured at different temperatures (see Fig. $1 \mathrm{a}$ and $\mathrm{b}$ ). The linewidths show the usual temperature dependent broadening of low frequency resonant modes which results from the anharmonicity. For both systems the integral absorption is nearly independent of temperature within the accuracy of the measurements. A remarkable effect is the shift of the $\mathrm{Tl}^{+}$resonances to higher frequencies with increasing temperature. Between 5.6 and $77^{\circ} \mathrm{K}$ this shift runs up to $20 \%$ for $\mathrm{CsI}: \mathrm{Tl}^{+}$and $9 \%$ for $\mathrm{CsBr}: \mathrm{Tl}^{+}$. This behaviour may be qualitatively understood by assuming that there is a positive self energy due to anharmonic processes, which increases with temperature and overcompensates the lowering of the frequency arising from the thermal expansion of the crystal $[5,17]$.

Earlier measurements of the resonant mode in CsI: $\mathrm{Tl}^{+}$under applied uniaxial stress did not show a splitting of the line. Therefore, in a previous paper [7] we concluded that thermal expansion does not influence this frequency. Recently improved measurements were performed with CsI crystals hardened by additional doping of about $10^{-3} \mathrm{~mol} \% \mathrm{Ca}^{2+}$ ions [18]. Thus uniaxial stress could be applied up to $21.6 \mathrm{Nmm}^{-2}$ which is about three times larger than the elasticity limit of pure or $\mathrm{Tl}^{+}$doped CsI crystals. Then a splitting of the mode could be observed. The same experiment was done with $\mathrm{CsBr}: \mathrm{Tl}^{+}$crystals. The temperatures of the samples were in both cases $6^{\circ} \mathrm{K}$. From these measurements it was found

$$
\begin{aligned}
& s\left(\Gamma_{1}^{+}\right) A\left(\Gamma_{1}^{+}\right)=(1.0 \pm 0.4) \times 10^{-3} \mathrm{~cm}^{-1} / \text { bar for } \mathrm{CsI}: \mathrm{Tl}^{+} \text {and } \\
& s\left(\Gamma_{1}^{+}\right) A\left(\Gamma_{1}^{+}\right)=(1.7 \pm 0.3) \times 10^{-3} \mathrm{~cm}^{-1} / \mathrm{bar} \text { for } \mathrm{CsBr}: \mathrm{Tl}^{+}
\end{aligned}
$$

Here $A\left(\Gamma_{1}^{+}\right)$is the linear anharmonic coupling coefficient, which describes the coupling of the resonant mode to a hydrostatic deformation $[2,19]$ and which is responsible for the shift of the frequency caused by thermal expansion, and $s\left(\Gamma_{1}^{+}\right)=s_{11}+2 s_{12}$ is the corresponding compliance of the crystal. To evaluate $A\left(\Gamma_{1}^{+}\right)$local compliances of the defect system should be used. However, such compliances are not yet known. Nevertheless, this result shows that at least the $\mathrm{Tl}^{+}$resonances will be affected by thermal expansion, hence, the self energy of the anharmonic processes must be still larger than the directly observed frequency shift. 


\section{Theory}

\subsection{Defect model}

The purpose of the following model calculation is to demonstrate the decoupling of the $\mathrm{In}^{+}$and $\mathrm{Tl}^{+}$impurities from the host lattices. We consider one substitutional impurity at a $\mathrm{Cs}^{+}$site in a lattice of $\mathrm{CsCl}$ type neglecting any interaction between the defects. Such an approximation is reasonable because of the low defect concentrations in the studied samples. A harmonic model is used involving the mass change and the change of the central force constants between the impurity and its eight nearest neighbours. The force constant change will be fitted to the resonance frequency. The theoretical concept of our calculation is reviewed in several articles $[3,20,21]$. Hence, only the basic equations will be stated whereas the results relevant for the $\mathrm{CsCl}$ structure will be given in more detail.

The frequencies of resonant modes are given by the solutions of the equation

where

$$
\operatorname{Re} D\left(\omega^{2}\right)=0 \text {, }
$$

$$
D\left(\omega^{2}\right)=\operatorname{det}|I+G \Lambda|
$$

is the denominator of the $T$-matrix (in matrix notation)

$$
T=\Lambda(I+G \Lambda)^{-1} \text {. }
$$

In this expression $I$ is the unit matrix, $G$ refers to the Green's function of the pure crystal, and $\Lambda$ is the local perturbation matrix containing the mass defect and the force constant change. The linewidth of a narrow resonance is given by

$$
\Delta \omega=\operatorname{Im} D\left(\omega_{\mathrm{r}}^{2}\right)\left\{\omega_{\mathrm{r}} \frac{\mathrm{d}}{\mathrm{d} \omega^{2}} \operatorname{Re} D\left(\omega_{\mathrm{r}}^{2}\right)\right\}^{-1},
$$

where $\omega_{\mathrm{r}}$ is the resonance frequency.

Considering only a change of the nearest neighbour interaction, $\Lambda$ becomes a $27 \times 27$ matrix with non-vanishing matrix elements corresponding to the subspace of the lattice affected by the impurity. The displacement vectors of the ions in this defect space form a reducible basis of the site symmetry of the impurity - in the present case the point group $O_{h}-$ which can be reduced to symmetry coordinates transforming according to the irreducible representations of the point group. The reduction is performed according to the same technique used to get the normal coordinates of a molecule. Then it is found that the irreducible representation $\Gamma$ of the group $\mathrm{O}_{h}$ appears in the defect space $n(\Gamma)$ times where

$$
\left.\begin{array}{llll}
n\left(\Gamma_{1}^{+}\right)=1, & n\left(\Gamma_{3}^{+}\right)=1, & n\left(\Gamma_{4}^{+}\right)=1, & n\left(\Gamma_{5}^{+}\right)=2, \\
n\left(\Gamma_{2}^{-}\right)=1, & n\left(\Gamma_{3}^{-}\right)=1, & n\left(\Gamma_{4}^{-}\right)=3, & n\left(\Gamma_{5}^{-}\right)=1 .
\end{array}\right\}
$$

The irreducible representations $\Gamma_{2}^{+}$and $\Gamma_{1}^{-}$do not enter the defect space [22]. The symmetry coordinates for the present impurity system are explicitly given in [23] and are used to determine the matrix elements of the defect matrix in 
the representation of these coordinates denoted by $\Lambda^{\Gamma}$ :

$$
\begin{aligned}
& \Lambda^{\Gamma_{1}^{+}}=\frac{\Delta f}{M_{-}} ; \quad \Lambda^{\Gamma_{3}^{+}}=\Lambda^{\Gamma_{4}^{+}}=\Lambda^{I_{3}^{-}}=\Lambda^{r_{\bar{\sigma}}^{-}}=\frac{\Delta g}{M_{-}} \\
& \Lambda_{j j^{\prime}}^{\Gamma_{4}^{-}}=\left(\begin{array}{ccc}
\frac{8(\Delta f+2 \Delta g)}{3 M_{+}}-\frac{\Delta M_{+}}{M_{+}} \omega^{2} & -\frac{8 \Delta f}{\left(24 M_{+} M_{-}\right)^{1 / 2}} & -\frac{8 \Delta g}{\left(12 \frac{\left.M_{+} M_{-}\right)^{1 / 2}}{M_{-}}\right.} \\
-\frac{8 \Delta f}{\left(24 M_{+} M_{-}\right)^{1 / 2}} & 0 & 0 \\
-\frac{8 \Delta g}{\left(12 M_{+} M_{-}\right)^{1 / 2}} & \frac{\Delta f}{M_{-}}
\end{array}\right), \\
& \Lambda_{j j^{\prime}}^{\Gamma_{5}^{+}}=\left(\begin{array}{cc}
\frac{\Delta f+2 \Delta g}{3 M_{-}} & \frac{\sqrt{2}(\Delta f+2 \Delta g)}{3 M_{-}} \\
\frac{\sqrt{2}(\Delta f+2 \Delta g)}{3 M_{-}} & \frac{2 \Delta f+\Delta g}{3 M_{-}}
\end{array}\right) .
\end{aligned}
$$

Here $M_{+}$and $M_{-}$are the masses of the positive and negative ions of the host crystal respectively, $\Delta M_{+}=M_{+}^{\prime}-M_{+}$is the mass defect and $\Delta f=f^{\prime}-f$, and $\Delta g=g^{\prime}-g$ are the changes of the nearest neighbour central and noncentral force constants. Primed letters refer to the defect crystal. $f$ and $g$ are related to the elements of the force constant matrices in the notation of Leibfried [24] as follows: $f=\alpha^{\prime \prime}+2 \gamma^{\prime \prime}$ and $g=\alpha^{\prime \prime}-\gamma^{\prime \prime}$. For the further discussion we only need $A_{j j^{\prime}}^{\Gamma_{4}^{-}}$, because only modes transforming according to the irreducible polar vector representation $\Gamma_{4}^{-}$interact with the radiation field by electric dipole absorption.

The Green's function of the pure crystal is evaluated using the Debye approximation with one Debye frequency given by Timmesfeld [25]. This approximation is reasonable because the resonant modes are lying in the very low frequency region of the phonon spectrum. According to Timmesfeld the Green's function of the unperturbed lattice has the form

where

$$
G_{\alpha \alpha^{\prime}}\left(\begin{array}{c}
l l^{\prime} \\
K K^{\prime}
\end{array} \mid \omega\right)=\delta_{\alpha \alpha^{\prime}} \frac{3\left(M_{K} M_{K^{\prime}}\right)^{1 / 2}}{\left(M_{+}+M_{-}^{-}\right) \omega_{\mathbb{D}}^{2}} g(d, \omega)
$$

and

$$
g(0, \omega)=1+\frac{\omega}{2 \frac{\omega_{\mathrm{D}}}{n}} \ln \left|\frac{1-\omega / \omega_{\mathrm{D}}}{1+\omega / \omega_{\mathrm{D}}}\right|+i \frac{\pi}{2} \frac{\omega}{\omega_{\mathrm{D}}}
$$

$$
\operatorname{Im} g(d, \omega)=\frac{\pi}{2} \frac{\sin \left(q_{\mathrm{D}} d \omega / \omega_{\mathrm{D}}\right)}{q_{\mathrm{D}} d} \text { for } \quad d \neq 0
$$

Here $l$ and $K= \pm$ label a unit cell and an ion in it respectively, $d=\mid \boldsymbol{R}\left(\begin{array}{l}l \\ K\end{array}\right)-$ $-\boldsymbol{R}\left(\begin{array}{c}l^{\prime} \\ K^{\prime}\end{array}\right) \mid$ is the equilibrium distance between two ions, $q_{\mathrm{D}}$ is the radius of a sphere in the reciprocal lattice having the same volume as the first Brillouin zone, $\omega$ is the frequency, and $\omega_{\mathrm{D}}$ the Debye frequency. The real part of $g(d, \omega)$ 
can be calculated by the Kramers-Kronig relation from $\operatorname{Im} g(d, \omega)$. With these expressions the Green's function in the representation of the $\Gamma_{4}^{-}$symmetry coordinates is found to have the following matrix elements:

$$
\begin{aligned}
& G_{11}=\frac{3 M_{+}}{f_{\mathrm{D}}} g(0, \omega) ; \\
& G_{12}=\frac{\left(24 M_{+} M_{-}\right)^{1 / 2}}{f_{\mathrm{D}}} g\left(\frac{\sqrt{3}}{2} a, \omega\right) ; \\
& G_{13}=\frac{\left(6 M_{+} M_{-}\right)^{1 / 2}}{f_{\mathrm{D}}} g\left(\frac{\sqrt{3}}{2} a, \omega\right) ; \\
& G_{22}=\frac{M_{-}}{f_{\mathrm{D}}}\{3 g(0, \omega)+g(a, \omega)+g(\sqrt{2} a, \omega)+3 g(\sqrt{3} a, \omega)\} ; \\
& G_{23}=\frac{4 \sqrt{2} M_{-}}{f_{\mathrm{D}}}\{g(a, \omega)+g(\sqrt{2} a, \omega)\} ; \\
& G_{33}=\frac{M_{-}}{f_{\mathrm{D}}}\{3 g(0, \omega)+5 g(a, \omega)+5 g(\sqrt{2} a, \omega)+3 g(\sqrt{3} a, \omega)\},
\end{aligned}
$$

where $f_{\mathrm{D}}=\left(M_{+}+M_{-}\right) \omega_{\mathrm{D}}^{2}$ and $a$ is the lattice constant.

When the matrices $\Lambda_{j j^{\prime}}^{\Gamma_{4}^{-}}$and $G_{j j^{\prime}}$ are substituted into equation (2) the denominator $D^{\Gamma_{4}^{-}}\left(\omega^{2}\right)$ corresponding to the $\Gamma_{4}^{-}$irreducible representation of the $T$ matrix turns out to be

$$
\begin{aligned}
D^{\Gamma_{4}}\left(\omega^{2}\right)= & 1+\Delta f\left\{\frac{3}{8} \frac{1}{M_{+}} G_{11}-\frac{16}{\left(24 M_{+} M_{-}\right)^{1 / 2}} G_{12}+\frac{1}{M_{+}} G_{22}\right\}-\frac{\Delta M_{+}}{M_{+}} \omega^{2} G_{11}- \\
& -\frac{\Delta f}{M_{-}} \frac{\Delta M_{+}}{M_{+}} \omega^{2} \operatorname{det}\left|G_{i j^{\prime}}\right|
\end{aligned}
$$

Evaluating this expression, the non-central part of the coupling $\Delta g$ has been neglected. Thus $\Lambda_{j j^{\prime}}^{\Gamma^{-}}$was reduced to a $2 \times 2$ matrix as can be seen from equation (6). This was obtained by choosing an appropriate orthonormal basis in the $\Gamma_{4}^{-}$subspace of which the mode labelled by $j=3$ affects only the non-central part of the coupling.

\subsection{Numerical results}

Now the resonance condition for optically active resonances $\operatorname{Re} D^{\Gamma_{4}^{-}}\left(\omega^{2}\right)=0$ was solved to get the change of the force constant $\Delta f(\omega)$ as a function of the frequency. Using this relation $\Delta f$ can be fitted to the observed resonance frequency. An effective low frequency nearest neighbour force constant $f$ of the unperturbed lattice is given by the solution of the resonance condition at zero frequency putting $\Delta f(\omega=0)=-f[20]$. For the present Debye model it turns out to be $f=0.276 f_{\mathrm{D}}$. Thus, the fractional change of the force constant $\Delta f / f$ can be calculated. The result is plotted in Fig. 2 versus the squared frequency $\omega^{2}$ within a frequency range reasonable for the Debye approximation.

The Debye frequencies determined by specific heat measurements are $\omega_{\mathrm{D}}=97 \mathrm{~cm}^{-1}$ for $\mathrm{CsBr}$ at $6^{\circ} \mathrm{K}$ and $\omega_{\mathrm{D}}=81 \mathrm{~cm}^{-1}$ for CsI at $5.6{ }^{\circ} \mathrm{K}$ [26]. These values were used to find the appropriate $\Delta f / f$ from Fig. 2 for the four defect 


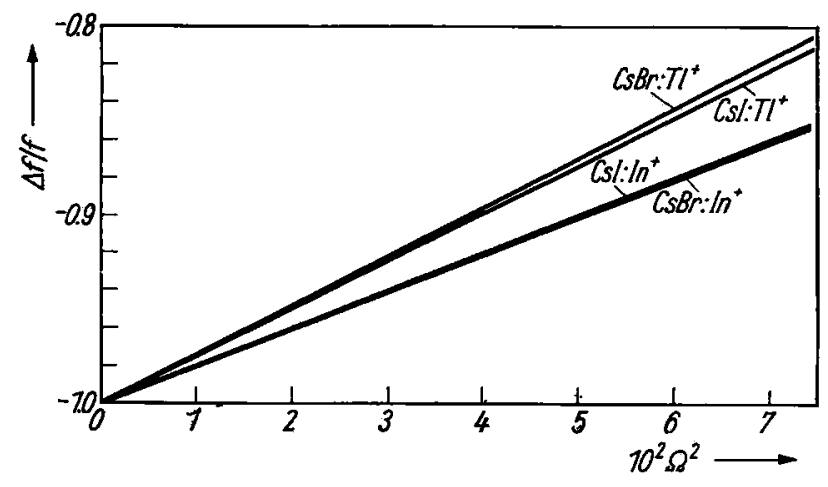

Fig. 2. Fractional change of the nearest neighbour central force constant $\Delta f / f$ versus normalized squared frequency $\Omega^{2}=\left(\omega / \omega_{D}\right)^{2}$

systems. Knowing $\Delta f$ the imaginary part of $D^{\Gamma_{4}^{-}}\left(\omega^{2}\right)$ (equation (11)) and the linewidth (equation (4)) can be evaluated. The resulting values of the force constant changes and the linewidths are given in Table 1 (c) and (d). The most weakly coupled defect is $\mathrm{In}^{+}$in $\mathrm{CsBr}$ whose force constant $f^{\prime}$ is only $2.4 \%$ of the original one. The ratio $f^{\prime} / f$ is nearly the same for both $\mathrm{Tl}^{+}$resonances: $7.9 \%$ for $\mathrm{CsBr}$ and $7.5 \%$ for CsI. In CsI :In ${ }^{+} f^{\prime} / f$ equals $4.4 \%$. The agreement between the experimental and theoretical linewidths is rather good for the resonances occurring in CsI but is bad for the resonant modes in CsBr. In this case the calculated linewidths are too narrow. This might be due to the shortcomings of the applied simple defect model especially due to the disregarding of the non-central forces. Calculations using the breathing shell model [10] for the host lattice phonons are performed by Martin and will be published later [27].

\section{Acknowledgements}

The authors wish to express their thanks to Prof. Dr. L. Genzel who stimulated the present work and to Dr. R. W. Alexander and Dr. K. Maier for many helpful discussions. They are also indebted to Dipl.-Phys. M. Schumacher who grew the crystals and carried out the chemical analysis. The support of the spectroscopic devices by the Deutsche Forschungsgemeinschaft is gratefully appreciated. The numerical calculations were performed at the computer IBM 7040 of the Rechenzentrum der Universität Freiburg i. Br.

\section{References}

[1] R. D. Ktrby, I. G. Nolt, R. W. Alexander, Jr., and A. J. Sievers, Phys. Rev. 168, 1057 (1968).

[2] I. G. Nolt and A. J. Sinvers, Phys. Rev. 174, 1004 (1968).

[3] M. V. Klein, Physics of Color Centers, Chap. 7, Ed. W. Beall Fowler, Academic Press, New York 1968.

[4] L. Genzel, Optical Properties of Solids, Chap. 15, Plenum Press, 1969.

[5] R. W. Alexander, A. E. Hughes, and A. J. Srevers, Phys. Rev. B1, 1563 (1970).

[6] R. D. Kirby, A. E. Hughes, and A. J. Srevers, Phys. Rev. B2, 481 (1970).

[7] L. Genzel, W. Prettl, and E. Siep, Optics Commun. 1, 28 (1969).

[8] E. B. Sandeld, Colorimetric Determination of Traces of Metals, Interscience Publishers, New York 1965.

[9] G. MAHLER, Diplomawork, University of Frankfurt, 1969.

[10] V. Nösslein and U. SChrödER, phys. stat. sol. 21, 309 (1967).

[11] G. Busse and G. HaIder, Optics Commun. 2, 45 (1970). 
[12] L. Paulina, J. Amer. Chem. Soc. 49, 765 (1927).

[13] J. R. Tessmann, A. H. Kahn, and W. Shockley, Phys. Rev. 92, 890 (1953).

[14] S. Masunaga, I. Morita, and M. Ishiguro, J. Phys. Soc. Japan 21, 638 (1966).

[15] K. MaIer, Abstracts of the 10th Europ. Congr. Molec. Spectr., Liège 1969.

[16] K. MaIen, Thesis, University of Frankfurt, 1970, and private communication.

[17] R. W. AleXander, Thesis, Cornell University, 1968.

[18] G. Busse and G. HaIdFr, to be published.

[19] G. Busse, W. Prettl, and L. Genzed, Phys. Letters (Netherlands) 27A, 438 (1968).

[20] G. Benedek and G. F. Nardelli, Phys. Rev. 155, 1004 (1967).

[21] G. Benedek and A. A. Maradudrn, J. Phys. Chem. Solids 29, 423 (1968).

[22] G. F. Koster, J. O. Dimmock, R. G. Wheeler, and H. Statz, Properties of the Thirty-Two Point Groups, The M.I.T. Press, Cambridge, Massachusetts 1963.

[23] W. LudwIG, Erg. exakt. Naturwiss. 35, 1 (1964).

[24] G. Leib Fried, Handbuch der Physik, Vol. VII/1, Springer-Verlag, 1955.

[25] K. H. TImmesfeLd, phys. stat. sol. 30, 73 (1968).

[26] M. Sorari, J. Phys. Soc. Japan 25, 421 (1968).

[27] T. P. Martin, to be published.

(Received February 8, 1971) 